\section{Braucht Deutschland ein neues Wirtschaftsmodell?}

Als die Deutschen 1990 die Einheit feierten, schien der Kapitalismus gesiegt zu haben, wie es Francis Fukuyama kurz zuvor mit seinem Essay zum „Ende der Geschichte“ proklamiert hatte. Mehr noch: Der Kollaps des Gegenmodells schien jenem paradigmatisch wirkenden Trend noch mehr Schub zu geben, die Balance sehr viel mehr wieder vom Staat weg in Richtung Markt zu verschieben - im tiefen Glauben, alle großen Probleme ließen sich am besten über das freie Spiel von Angebot und Nachfrage regeln. Damals war die Hochzeit für das Privatisieren staatlicher Einrichtungen. Es begann ein Jahrzehnt der Hyper-Finanzglobalisierung. Und es ging fast nur darum, wie sich noch mehr Regeln und Grenzen abbauen lassen; wie Einkommen wieder stärker gespreizt werden, um vermeintlichen Leistungsträgern mehr Anreize zu geben - deren Früchte über Trickle-Down dann am Ende alle erreichen sollten. Und wie der Staat Hilfsleistungen abbaut. Motto: Eigenverantwortung. 30 Jahre später scheint eben dieser Kapitalismus in der Glaubwürdigkeitskrise. Nach einer Umfrage der Beratungsagentur Edelman sagen weltweit $56 \%$ der Befragten, dass der Kapitalismus in seiner heutigen Form nicht geeignet sei, die großen Probleme der Menschheit zu lösen. Die Kritik wird lauter. Auch der Papst wettert gegen die Macht der Märkte.

Nur ein Modephänomen, wie manche Liberale sagen? Vielleicht. Nur spricht heute viel für eine tatsächlich grundlegendere Krise. Und wenn das stimmt, sollte die Frage eher sein, was genau dahintersteckt - und wie diese Krise zu beheben ist. Dafür eine offene Plattform zu bieten, ist Ziel des Forum New Economy, das seit Oktober 2019 Forschung und Praxis zusammenbringt. Und: um die Relevanz all dieser Fragen für Deutschland auszuloten, haben wir kurz vor dem 30. Jahrestag der Einheit über drei Tage das Für und Wider neuer ökonomischer Antworten diskutiert: in einem Gespräch zwischen Nobelpreisträger Joseph Stiglitz und Bundesfinanzminister Olaf Scholz; durch Keynotes von Thomas Piketty und Jens Spahn, der als Lehre aus der Corona-Krise für ein neues Staatsverständnis plädiert; und durch die Präsentation exklusiver Studien rund um die großen Grundsatzfragen etwa von Mariana Mazzucato, Michael Hüther, Jens Südekum, Achim Truger und anderen. Einen Teil der Beiträge zur Frage nach einem „neuen deutschen Modell“ dokumentiert das Zeitgespräch in diesem Heft.

Dass es in diesen Zeiten in Deutschland ebenso Bedarf gibt, die Balance zwischen Staat und Markt neu auszutarieren, lassen Umfragen auch hier erahnen. Nach einer Forsa-Umfrage im Auftrag des Forums sagen immerhin knapp $30 \%$ der Menschen im Land, dass das Wirtschaftssystem, so wie es heute funktioniert, grundlegend erneuert werden sollte - mehr als $60 \%$ geben an, dass es zumindest "in einigen Bereichen verbessert" werden sollte. Und: Nur $5 \%$ sehen keinen Bedarf. Jeder Zweite sagt, dass das Versprechen des sozialen Ausgleichs nicht mehr funktioniert. Da ist es erst einmal egal, ob die Einschätzung berechtigt wirkt oder nicht - all das drückt eine tiefere Vertrauenskrise aus, die womöglich dann doch auch ihre Gründe hat. Statt privater Investitionsschübe, wie sie die Anhänger der markliberalen Wende einst versprachen, gibt es heute eine dramatische Unterausstattung öffentlicher Infrastruktur. Statt für Effizienz zu stehen, haben Finanzmärkte immer wieder gezeigt, wie viel selbstreferenzielle Instabilität sie erzeugen können - bis hin zur größten Finanzkrise seit den 1930er Jahren. Und die Globalisierung erwies sich als etwas, das in den reicheren Ländern eben doch auch gravierende Nebenfolgen hat - wie sich an den dramatischen Folgen des Niedergangs ganzer Industrieregionen in

C Der/die Autor(en) 2020. Open Access: Dieser Artikel wird unter der Creative Commons Namensnennung 4.0 International Lizenz (https://creativecommons.org/licenses/by/4.0/deed.de) veröffentlicht.

Open Access wird durch die ZBW - Leibniz-Informationszentrum Wirtschaft gefördert.

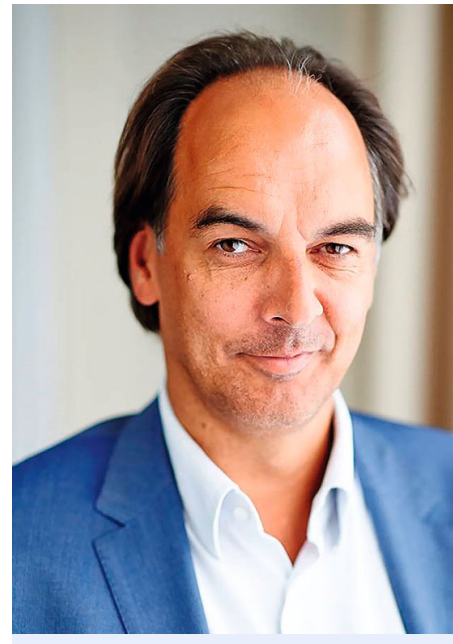

Thomas Fricke ist Direktor des Forum New Economy und Kolumnist bei Spiegel Online. 
den USA und Großbritanniens gezeigt hat, in denen viele dann für Trump und Brexit wählten. All das mag in den USA und Großbritannien, wo der Glaube an die heiligen Märkte weit exzessiver ausgelebt wurde, noch ein Stück dramatischer sein als bei uns. Da sind Vermögen und Einkommen gesellschaftlich dramatisch auseinandergedriftet - und das Trickle-Down hat eben nicht funktioniert. Wie die Auswertungen von Charlotte Bartels zeigen, deutet auch für Deutschland immer mehr darauf hin, dass Vermögen und Einkommen heute sehr viel stärker auseinanderliegen als vor 1990 - auch wenn sich der Befund je nach Indikator seit 15 Jahren hier und da nicht weiter verschlimmert hat. Die Frage ist ja eher: Hätte in den langen Jahren des deutschen Wirtschaftswachstums nicht das viel beschworene Trickle-Down zu stark sinkender Ungleichheit führen müssen? Was die Ungleichheit von Vermögen angeht, hat zwar der Immobilienpreisanstieg seit 2012 dazu geführt, dass der Abstand zwischen der Mittelschicht, in der es viele Immobilieneigentümer gibt, und den Top-Vermögenden kleiner geworden ist. Der Abstand zwischen den Top-Vermögenden und der unteren Hälfte der Bevölkerung hat sich drastisch vergrößert. Entscheidend ist, die Treiber solcher Entwicklungen besser identifizieren zu können - um möglichst effizient zu bestimmen, was solche Trends stoppen könnte.

Es gibt etliche wirtschaftliche Tätigkeiten, aus denen sich der Staat zurückhalten sollte, klar. Wie in den USA und Großbritannien hat auch in Deutschland das Dogma von der vermeintlichen Überlegenheit des Marktes etliche Schäden mit sich gebracht. Dazu gehört, dass zu wenig öffentlich investiert wurde. Auch das ist Teil der Vertrauenskrise des marktliberalen Paradigmas, wie Michael Hüther und Jens Südekum in ihrem Beitrag darlegen. Zu einem neuen Modell müsse gehören, die Schuldenbremse so zu reformieren, dass wichtige Zukunftsinvestitionen nicht mehr kurzfristigen Haushaltsnöten geopfert werden. Was für die öffentliche Infrastruktur gilt, gilt ähnlich für jene Industriepolitik, die in Deutschland lange als tabu galt - zumindest in den ordnungspolitischen Sonntagsreden. Wie Mariana Mazzucato und Rainer Kattel darlegen, steht Deutschland modellhaft dafür, wie der Staat vor allem die Verbreitung neuer Technologien befördert hat - und das durchaus aktiv. Doch wenn es um die großen Herausforderungen unserer Zeit wie den Klimawandel oder die Digitalisierung geht, stoßen wir an Grenzen. Eine so große Transformation, wie sie derzeit die Mobilitätssysteme erfasst, gelingt nicht einfach über den offenen Wettbewerb der Technologien. Da braucht es einen Staat, der die Richtung vorgibt. Deutschland habe heute die Chance, seine Stärke in der Diffusion von Technologien mit einem neuen Verständnis von missionsorientierter Politik zu verbinden, so Mazzucato und Kattel. Was wiederum nahtlos zu einer weiteren Baustelle der Modellerneuerung führt - und zur Frage, ob und wie es Deutschland schaffen kann, die eigene Abhängigkeit vom Exportgeschäft zu lösen, in einer Zeit, in der die Globalisierung ins Stocken geraten und China nicht mehr der dynamische Importeur ist, der es gerade für deutsche Exporteure in den 2000er Jahren war.

Wie Achim Truger und seine Ko-Autoren darlegen, sind Deutschlands viel kritisierte Überschüsse im Außenhandel ohnehin eher deshalb entstanden, weil das Wachstum der deutschen Importnachfrage stark nachgelassen hat. Was nahelegt, dass der Abbau der Überschüsse nicht durch weniger Export, sondern mehr Import kommen - und die Deutschen mehr Geld ausgeben sollten. Die Frage ist, ob das ohne institutionelle Veränderungen wie die Stärkung der Tarifpartner geht, über die gewährleistet würde, dass Löhne und Einkommen dynamischer steigen - ohne dass dies die Industrie existenziell gefährdet. All das heißt nicht, dass der Staat jetzt alles machen soll und der Kapitalismus abgeschafft gehört. Und es heißt auch nicht, dass bei jeder einzelnen der Herausforderungen schon der Weisheit letzter Schluss gefunden ist. Es geht nur darum, in einer Zeit von Klimawandel, sozialen Spaltungen und Demokratiekrisen eine Schlagseite im Wirtschaftssystem zu korrigieren, die vor lauter Siegesgewissheit nach dem Fall der Mauer vor drei

Thomas Fricke

Forum New Economy thomas.fricke@newforum.org
Jahrzehnten entstanden ist und zu einem Überschießen des Marktglaubens als Leitmotiv für die Wirtschaftspolitik geführt hat - und dessen Schäden zur heutigen Vertrauenskrise der liberalen Gesellschaften beitragen. Eine neue Balance muss gefunden werden. 